\title{
Prioritized Soft Constraint Satisfaction: A Qualitative Method for Dynamic Transport Selection in Heterogeneous Wireless Environments
}

\author{
Heidi R. Duffin \\ Michael A. Goodrich \\ mike@cs.byu.edu \\ Charles D. Knutson \\ knutson@cs.byu.edu
}

Follow this and additional works at: https://scholarsarchive.byu.edu/facpub

Part of the Computer Sciences Commons

\section{Original Publication Citation}

Heidi R. Duffin, Charles D. Knutson, and Michael A. Goodrich. "Prioritized Soft Constraint Satisfaction: A Qualitative Method for Dynamic Transport Selection in Heterogeneous Wireless Environments." Proceedings of the IEEE Wireless Communication and Networking Conference (WCNC 24), Atlanta, Georgia, March 21-25, 24.

\section{BYU ScholarsArchive Citation}

Duffin, Heidi R.; Goodrich, Michael A.; and Knutson, Charles D., "Prioritized Soft Constraint Satisfaction: A Qualitative Method for Dynamic Transport Selection in Heterogeneous Wireless Environments" (2004). Faculty Publications. 450. https://scholarsarchive.byu.edu/facpub/450 accepted for inclusion in Faculty Publications by an authorized administrator of BYU ScholarsArchive. For more information, please contact ellen_amatangelo@byu.edu. 


\title{
Prioritized Soft Constraint Satisfaction: A Qualitative Method for Dynamic Transport Selection in Heterogeneous Wireless Environments
}

\author{
Heidi R. Duffin, Charles D. Knutson, Michael A. Goodrich \\ Computer Science Dept. \\ Brigham Young University \\ Provo, UT 84602 \\ \{hduffin,knutson,mike\}@cs.byu.edu
}

\begin{abstract}
This paper presents Prioritized Soft Constraint Satisfaction (PSCS), a novel approach to selecting the "best" transport in dynamic wireless transport switching systems. PSCS maintains a satisfying connection to another endpoint by choosing transports based on a user-established range of preferences and priority for criteria such as speed, power, range and cost. Additionally, feedback is provided regarding tradeoffs among the criteria, thus enabling the user to adjust inputs according to the capabilities of the system. We also recommend guidelines for setting preferences and priorities.
\end{abstract}

\section{INTRODUCTION}

It is increasingly common for mobile devices to be equipped with multiple wireless transceivers such as IrDA infrared, Bluetooth, wireless local area network (WLAN), or cellular. Such intra-device heterogeneity can be exploited to optimize link quality via intelligent, dynamic transport selection independent of session layer protocols or user applications. In this paper, link quality is optimized through 1) the ability to switch between radically different transports through the Quality of Transport (QoT) protocol described in section II and 2) a novel approach to decision making which we call Prioritized Soft Constraint Satisfaction (PSCS).

The rest of this paper is organized as follows. Section II relates similar research in the multi-transport domain. Section III presents PSCS, with details on the algorithm and supporting methods given in sections IV and V. Section VI examines user profiles and inputs to PSCS. Section VII presents conclusions.

\section{PRIOR WORK}

A number of research projects have examined heterogeneous connection capabilities, though most have presumed a single usage model (normally Internet or LAN access) and a single transport protocol (typically TCP/IP).

The MosquitoNET project at Stanford University studied continuous Internet connectivity to mobile hosts [1]. This project took advantage of "the best connection available," whether wired (Ethernet) or wireless (radio modem), but did not discuss a method for choosing that connection.

The University of Bradford, the United Kingdom, introduced a fuzzy logic approach as the selection algorithm for choosing between terrestrial and satellite networks based solely on the tradeoff between cost and quality [2].

The BARWAN project at the University of California at Berkeley explored the use of vertical handoffs in wireless overlay networks as a mechanism for intelligently and dynamically maintaining an active TCP/IP connection to a network infrastructure [3]. BARWAN researchers' first approach to decision making was a hierarchy in which the transport with the smallest footprint was assumed to be the fastest and hence the best. This model is simplistic in that the smallest footprint is not always the fastest (for example, Bluetooth has a smaller footprint than $\mathrm{WiFi}$, but much less speed) and a faster connection is not always better (for example, $\mathrm{WiFi}$ is faster than IrDA, but draws much more power).

The BARWAN group improved on this early method by allowing users to influence transport selection with a policybased scheme [4] in which the user was asked to assign a percentage value to the criteria of speed, power and cost with the total equaling $100 \%$. The more important criteria were given a higher percent of the total. This method has several shortcomings. First, the performance capabilities of the device are not indicated - when speed is set at $41 \%$ the user doesn't know if that number represents $54 \mathrm{Mbps}$, or $115 \mathrm{Kbps}$. Second, the tradeoffs are not reflected. For instance, physical laws make it unfeasible to meet requests for high range and low power simultaneously. However, the user has no idea about this fundamental tradeoff and therefore can not adequately choose between the two criteria. Finally, no feedback is provided. Once the numbers are input, the user has no idea about the kind of performance they are achieving.

In the Mobile Computing Laboratory at Brigham Young University we explored utilizing multiple transports simultaneously to maximize connection throughput to a peer device via inverse multiplexing [5]. However, this research did not provide decision making regarding appropriate situations in which to use the increased speed potential.

This paper describes decision making extensions to Quality of Transport (QoT), a system that facilitates dynamic and transparent transport switching [6] [7]. QoT is a protocol layer residing between the transport and session layers of the OSI network model. It provides a bridge between the upper layers of any transport protocol stack and the lower layers of any other transport protocol stack. Thus traffic from any session protocol common to two devices can be routed over any transport shared by the two devices (see Figure 1).

\section{PRIORITIZEd Soft Constraint SATISFACTION}

For the average user, choosing between transports does not revolve around the transports themselves, but rather on a 


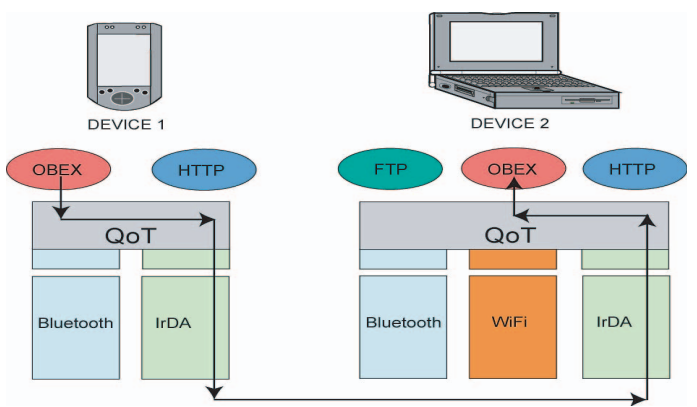

Fig. 1. Multi-transport data routing using QoT.

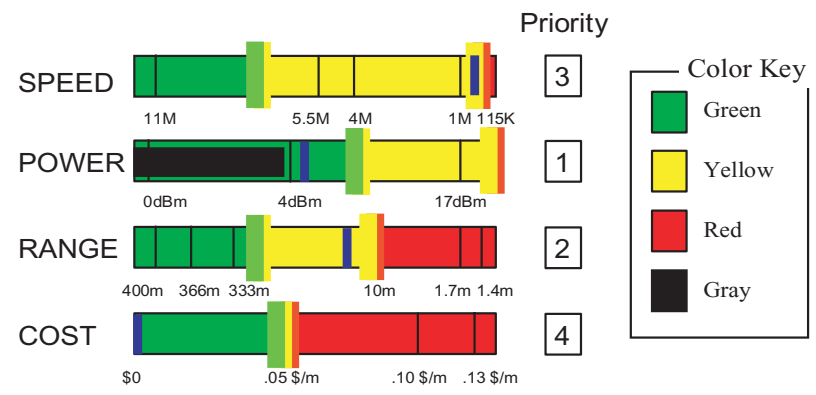

Fig. 2. An example of a user profile for PSCS.

set of descriptive criteria. Many decision making approaches consider battery power, bandwidth and network cost to be among the most important criteria [2] [4] [8] [9]. The list could be expanded to include signal range, signal quality, latency, error rate, service priority, user activity, jitter, connection time, security, interference and data type. Decision making in a transport switching system should take user input on criteria such as these and translate that input into a decision on a single preferred transport.

This research introduces Prioritized Soft Constraint Satisfaction (PSCS) for decision making. The graphical user interface (GUI) for PSCS, depicted in Figure 2, receives user input on criteria for speed, power, range and cost. These four criteria were selected because they are highly applicable to most users, although other criteria could be included. Each criterion is presented as a 2-slider selection bar with an accompanying priority number. Each bar shows a set of possible values for that criterion and relevant points are labelled. The user can move the green-yellow slider to control the range of values that are desirable (the green area) or acceptable (the yellow area) and the yellow-red slider to control the ranges that are acceptable or unacceptable (the red area), using the classic color scheme for go, caution and stop. The priority numbers indicate the criteria values that are most important to attain, enabling the device to meet preferences on more important criteria by sacrificing preferences on less important criteria when necessary. The slider values and priority numbers together make up the user profile, which PSCS uses to determine the most desirable transport based on user preferences.

PSCS addresses the shortcomings of previous decision making methods in three areas. First, the user can see the performance options of the device. For instance, Figure 2 shows a SPEED range from $115 \mathrm{Kbps}$ to $11 \mathrm{Mbps}$. PSCS also provides a pop-up menu to translate the data points into more intuitive values based on a download standard such as a 3-minute $(40 \mathrm{Mb})$ movie trailer. In this example, speed values are changed from Mbps to time, power is changed from $\mathrm{dBm}$ to battery life remaining and cost is changed from dollars per minute to a predicted total charge.

Second, tradeoffs between criteria are shown as gray zones that indicate options no longer available based on the position of the red sliders. Figure 2 shows an instance in which the red slider on RANGE has caused a gray zone to appear on POWER. The gray zones aid the user in developing the knowledge to choose between tradeoffs. We discuss gray zones in greater detail in section $\mathrm{V}$.

Finally, feedback is provided through blue lines indicating the performance of the current connection. Using this feedback the user may adjust his/her profile on-the-fly during a connection to attempt better performance in certain areas. In some cases a criterion may not be measurable. Range, for instance, is difficult to determine unless a technology like global positioning is utilized by both endpoints of the connection.

Performance measurement is also used to decide when to switch transports. If the measurement falls into a lower range of constraints for a sufficient time period, then QoT may attempt to switch transports if one is available with better performance potential. For instance, an $802.11 \mathrm{~b}$ connection which promises an $11 \mathrm{Mbps}$ data rate may only yield $2 \mathrm{Mbps}$. If this reduced throughput is still satisfying to the user (the measurement line does not cross constraint boundaries), then QoT remains on that transport. Otherwise QoT might switch to a $4 \mathrm{Mbps}$ IrDA connection, attempting to achieve greater than 2 Mbps throughput.

\section{The PSCS Algorithm}

PSCS uses negotiation to compute a preferred transport. If no transport meets all the desirable settings of the user profile, then PSCS compromises on the preferences of the lowest priority criteria first until a satisfying solution is reached. PSCS utilizes a binary tree to capture a hierarchical ordering of the criteria established by the user in the GUI. The highest priority criterion is the root node of the tree and lower priority criteria form subsequent nodes. The left and right branches of each node are taken from the desirable and acceptable ranges for the criterion respectively and are referred to as the green and yellow branches. (Note that the values for the yellow branch include the values for the green branch.)

The algorithm begins with a list of all transports on the device. The tree is traversed in a depth-first manner, eliminating transports from the list that do not meet the requirements of the current path and backtracking when the list is empty. When a leaf is reached that contains one or more transports, the algorithm halts.

As an example, we begin with a transport list containing the IrDA, Bluetooth and WiFi transports from Figure 4. The binary tree in Figure 3 represents the PSCS computation for the profile of Figure 2. Power is the first node. The green 


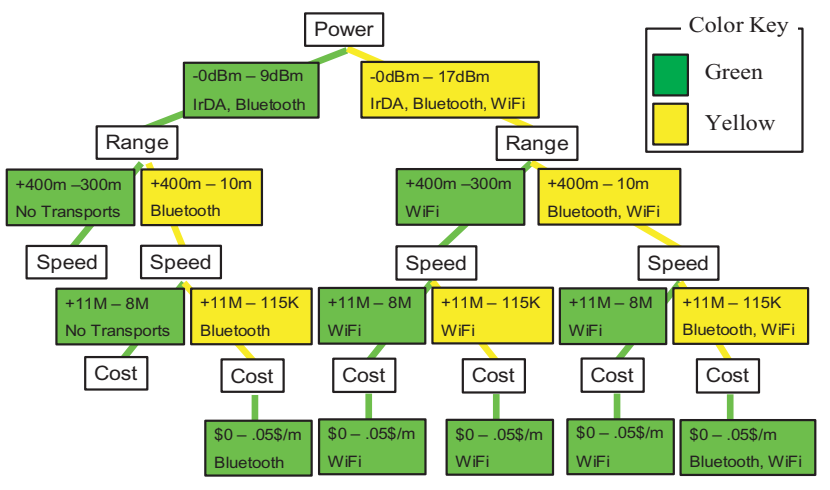

Fig. 3. An example of the binary priority tree used by the PSCS algorithm.

branch under power ( 0 to $9 \mathrm{dBm}$ ) restricts $\mathrm{WiFi}$ from the list. The green branch under range $(400$ to $300 \mathrm{~m})$ restricts IrDA and Bluetooth from the list. Since the list is empty, the yellow branch under range (400 to $10 \mathrm{~m}$ ) is expanded, leaving only Bluetooth in the list. Next, the green branch under speed (11 to $8 \mathrm{Mbps}$ ) is expanded and again produces an empty list. The yellow branch under speed (11 to .115 Mbps), however, keeps Bluetooth in the list as does the green branch under cost ( $\$ 0$ to $\$ .05 / \mathrm{min}$ ). A leaf has been reached with at least one transport in the list, Bluetooth, which is the transport selected.

When the first phase of the algorithm halts, if there is only one transport in the list, that transport is used. If more than one transport is in the list, the tie is broken by returning to the top priority and selecting the transport that performs best for that priority. If the tree is completely traversed and there are no transports in the list, then no connection is established. In this case the user has set the unacceptable ranges in such a way as to eliminate all transports from consideration. Fortunately, this is indicated to the user by the gray zones. When the gray zones completely cover the green and yellow ranges on any criteria, no transport will pass the user profile and the sliders should be adjusted.

\section{THE GRAY ZONES}

Decision making criteria are often interrelated, either naturally by physical laws or artificially by a transport vendor or connection service provider. The gray zones allow us to capture these relationships as limitations on potential performance. As an example, in Figure 2 the red slider on RANGE is set to $9.9 \mathrm{~m}$. This means that any range less than $10 \mathrm{~m}$ is unacceptable. For the transports in Figure 4 a power output of at least $4 \mathrm{dBm}$ is required to achieve at least a $10 \mathrm{~m}$ range. This is indicated by the gray zone on POWER, which shows that any power setting less than $4 \mathrm{dBm}$ is unattainable. The user may choose to adjust the slider settings on POWER to accommodate this new information.

Although a connection may exhibit poorer performance than the gray zones indicate, this limitation cannot be known at the time of establishing the user profile. For instance, although range is affected by interference, there is no way to predict the amount of interference that will exist at the time of the connection. Therefore the gray zones can only indicate a bound on performance and not precise per-connection limitations.

\section{A. Factors Affecting Criteria}

Various factors influence each of the four criteria: speed, power, range and cost. Moreover, the criteria affect each other in predictable ways.

Speed. Complex encoding schemes are sometimes used to produce high data rates. Such encoding schemes typically require a strong, clear signal to achieve good throughput, which is realized via higher output power. Additionally, a signal is less likely to encounter interference when it traverses shorter distances.

Power. Low output power is beneficial since it increases battery life, creates less interference for other signals, and reduces radiation exposure to the user. For these reasons, the maximum allowable radiated power is regulated by the Federal Communications Commission (FCC). Low output power produces weaker signals, thus lower data rates must be used to achieve acceptable throughput. However, weaker signals can be effective when the communicating devices are proximate. In fact, many wireless vendors build power adjustment capabilities into their transceivers and controllers so that when the detected signal is too strong or too weak the receiver may inform the transmitter to decrease or increase power.

Range. High range allows greater mobility and less overhead for handoffs. Range is boosted by using low frequencies, and antennas with high gain and sensitivity. Using a longer range generally requires a lower data rate since fewer bits per packet means there is less chance of failed communication at a specified BER (bit error rate). High output power also increases range by producing strong signals which can travel farther and still overcome interference.

Cost. Infrastructure-based transports, such as cellular and satellite (and sometimes WiFi hotspots), are regulated by cost plans established by a service provider. In some cases users pay more for a faster connection, or a wider coverage area, but this relationship may not always hold. When charge is regulated by data size (e.g., \$.002/byte) the speed of the transport does not matter, whereas for a per time charge (e.g., $\$ .10 /$ minute) a faster transport is better. From the perspective of QoT, if the user is subscribed to a monthly plan with unlimited data access, this is equivalent to a $\$ 0$ cost.

\section{B. Theoretical Limits}

Speed, power and range can all be related mathematically. The following equations provide absolute limitations on one criterion based on conditions for other criteria.

The equation for Shannon's Law provides a maximum limit for data rate as a function of output power.

$$
C=B * \log _{2}(S N R+1)
$$

Where:

- $\mathrm{C}$ is the capacity of the channel in Mbps.

- $\mathrm{B}$ is the bandwidth in $\mathrm{MHz}$.

- $\mathrm{SNR}$ is the signal to noise ${ }^{1}$ ratio in $\mathrm{dB}$.

${ }^{1}$ The noise level in normal conditions without any competing WLAN on the frequency and without industrial noise is generally around $-100 \mathrm{dBm}$. 
PSCS can use this relationship to set a gray zone on SPEED based on the setting of the red slider for POWER.

Similarly, by solving Equation 1 for power we can determine the minimum power output required to achieve a specified data rate. PSCS can use this to set the gray zone on POWER when the red slider is set on SPEED.

The equation relating range to power is taken from well known equations for link budget and path loss in free space ${ }^{2}$.

$$
D=10^{\left(P_{T X}-S_{R X}-92.4-20 * \log _{10} F\right) / 20}
$$

Where:

- $\mathrm{D}$ is the distance in $\mathrm{km}$.

- $P_{T X}$ is the output power of the transmitter in $\mathrm{dBm}$.

- $S_{R X}$ is the sensitivity of the receiving antenna in $\mathrm{dBm}$.

- $\mathrm{F}$ is the frequency of the transmission (center of band) in GHz.

This equation provides the theoretical maximum distance attainable from a given power output, transmitting frequency and receiving antenna sensitivity ${ }^{3}$. PSCS can use this relationship to set the gray zone on RANGE based on the setting of the red slider for POWER.

Equation 2 can also be used to determine the minimum power output required to achieve a specified range. PSCS can use this to set the gray zone on POWER when the red slider is set on RANGE.

\section{Realistic Limits}

Although the theoretical equations provide PSCS with the necessary information to set the gray zones, considering details such as encoding schemes, packet sizes, window sizes, duplex modes, signal blockage, power lost to cabling or heat, and so on, it is apparent that the best practical performance for any given transport will fall below the theoretical optimum. Therefore, we have devised a set of realistic equations in connection with transport specifications that produce a much tighter bound on performance limitations.

In explaining the realistic equations, we will use an example taken from a typical mobile device. Figure 4 shows the specification data for an HP iPAQ Pocket PC 5400 Series. The iPAQ has built-in IrDA, Bluetooth, and WiFi 802.11b, with performance capabilities as listed.

Each entry in the specification data can be written as the 4-tuple:

$$
\left(D_{i}, P_{i}, R_{i}, T_{i}\right)
$$

Where:

- $D_{i}$ is the data rate in Mbps.

- $P_{i}$ is the output power in $\mathrm{dBm}$.

- $R_{i}$ is the range in meters.

- $T_{i}$ is the transport name.

\footnotetext{
${ }^{2}$ This equation assumes no power loss from cabling and zero gain from antennas.

${ }^{3}$ The receiving antenna sensitivity will not be known prior to connection. However, we can assume that the transports will be similar on both sides of the connection. Therefore we use the transmitting transport's own receiving sensitivity as an estimate for this value.
}

\begin{tabular}{|c|c|c|c|}
\hline & Speed & Power & Range \\
\hline $802.11 \mathrm{~b}$ & $11 \mathrm{M}$ & $17 \mathrm{dBm}$ & $333 \mathrm{~m}$ \\
\cline { 2 - 4 } & $5.5 \mathrm{M}$ & $17 \mathrm{dBm}$ & $366 \mathrm{~m}$ \\
\cline { 2 - 4 } & $1 \mathrm{M}$ & $17 \mathrm{dBm}$ & $400 \mathrm{~m}$ \\
\hline IrDA & $4 \mathrm{M}$ & $0 \mathrm{dBm}$ & $1.4 \mathrm{~m}$ \\
\cline { 2 - 4 } & $115 \mathrm{~K}$ & $0 \mathrm{dBm}$ & $1.7 \mathrm{~m}$ \\
\hline Bluetooth & $723 \mathrm{~K}$ & $4 \mathrm{dBm}$ & $10 \mathrm{~m}$ \\
\hline
\end{tabular}

Fig. 4. Specification data for the HP iPAQ Pocket PC 5400 series.

As an example, IrDA has two entries, namely (4, 0, 1.4, IrDA) and (.115, 0, 1.7, IrDA). The set of entries for a device is the vector:

$$
\left[\left(D_{1}, P_{1}, R_{1}, T_{1}\right),\left(D_{2}, P_{2}, R_{2}, T_{2}\right), \ldots,\left(D_{n}, P_{n}, R_{n}, T_{n}\right)\right]
$$

The HP iPAQ has six entries for its three transports.

PSCS uses the specification entries in two instances. First, the PSCS algorithm described in section IV uses these entries to decide if a transport meets the constraints of the user profile. Second, the gray zones in PSCS are set from this data, using the following realistic equations:

$$
\begin{aligned}
& D_{\max }=\operatorname{argmax}_{D}\left(D_{i}, P_{i}\right): P_{i}<P_{L} \\
& D_{\max }=\operatorname{argmax}_{D}\left(D_{i}, R_{i}\right): R_{i}>R_{L} \\
& P_{\text {min }}=\operatorname{argmin}_{P}\left(D_{i}, P_{i}\right): D_{i}>D_{L} \\
& P_{\min }=\operatorname{argmin}_{P}\left(D_{i}, R_{i}\right): R_{i}>R_{L} \\
& R_{\max }=\operatorname{argmax}_{R}\left(D_{i}, R_{i}\right): D_{i}>D_{L} \\
& R_{\max }=\operatorname{argmax}_{R}\left(P_{i}, R_{i}\right): P_{i}<P_{L}
\end{aligned}
$$

Where:

- $D_{\max }, P_{\min }$ and $R_{\max }$ are the maximum or minimum achievable data rate, power and range respectively.

- $D_{i}, P_{i}$ and $R_{i}$ are the specification data for entry $i$ on data rate, power and range respectively.

- $D_{L}, P_{L}$ and $R_{L}$ are the user-specified limits taken from the red sliders on SPEED, POWER and RANGE respectively.

As an example, consider Figure 2, which shows a minimum required power resulting from a minimum demanded range. This gray zone is derived from Equation 8 in the following manner. First, the RANGE red slider is set to $9.9 \mathrm{~m}\left(R_{L}=9.9\right.$ $\mathrm{m})$. The iPAQ specification entries with range $R_{i}$ greater than $R_{L}$ are $[(1,4,10$, Bluetooth), (11, 17, 333, WiFi), (5.5, 17, 366, WiFi), (1, 17, 400, WiFi)]. The minimum power value of this set is $4 \mathrm{dBm}\left(P_{\min }=4 \mathrm{dBm}\right)$. The gray zone covers the 


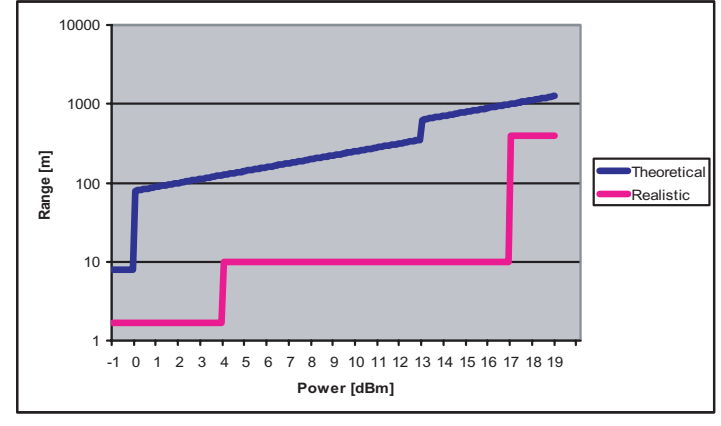

Fig. 5. Comparison of the theoretical and realistic limitations for range as a function of power.

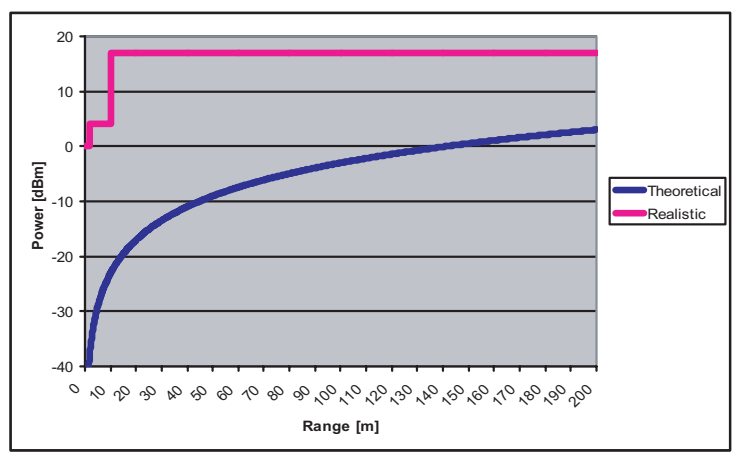

Fig. 6. Comparison of the theoretical and realistic limitations for power as a function of range.

POWER bar from its leftmost side up to, but not including, 4 $\mathrm{dBm}$.

Similar equations are given for each criterion relationship. When multiple equations come into play, the most restrictive value is used. Equations exist for the cost relationship as well, since cost can be related to the other criteria in this manner. However, since the transports on the iPAQ are all peer-to-peer transports, the cost equations are not presented.

Figures 5 and 6 compare two theoretical equations to the realistic ones. Notice in these graphs that the realistic equations follow the same trend as the theoretical ones. Also, the realistic equations produce a much tighter bound than the theoretical ones, thus providing the user with a clearer understanding of the system's capabilities. For instance, in Figure 5, for a power output of $2 \mathrm{dBm}$, the theoretical equation limits the potential range to $100 \mathrm{~m}$, while the realistic equation places the maximum achievable range at $1.7 \mathrm{~m}$ ! At $18 \mathrm{dBm}$, both equations set a higher maximum on range. The theoretical limit is $1121 \mathrm{~m}$, but the realistic limit is still tighter at $400 \mathrm{~m}$. The divergence in the comparison results from the theoretical equation accounting for all potential operating modes of a transport, whereas the realistic equations result from a particular instantiation of a transport set within a particular device.

\section{Establishing User Profiles}

This section examines the results of using PSCS, including 1) a perspective on how subtle changes to the user profile can

\begin{tabular}{|l|c|c|c|c|c|c|}
\hline & $\begin{array}{l}\text { I=Speed } \\
\text { 2=Power } \\
\text { 3=Range }\end{array}$ & $\begin{array}{c}\text { 1=Speed } \\
\text { 2=Range } \\
\text { 3=Power }\end{array}$ & $\begin{array}{c}\text { 1=Power } \\
\text { 2=Speed } \\
\text { 3=Range }\end{array}$ & $\begin{array}{c}\text { 1=Power } \\
\text { 2=Range } \\
\text { 3= Speed }\end{array}$ & $\begin{array}{c}\text { 1=Range } \\
\text { 2= Speed } \\
\text { 3=Power }\end{array}$ & $\begin{array}{c}\text { 1=Range } \\
\text { 2=Power } \\
\text { 3= Speed }\end{array}$ \\
\hline Slider Setting 1 & IrDA & $802.11 \mathrm{~b}$ & IrDA & Bluetooth & $802.11 \mathrm{~b}$ & Bluetooth \\
\hline Slider Setting 2 & IrDA & IrDA & IrDA & Bluetooth & Bluetooth & Bluetooth \\
\hline Slider Setting 3 & IrDA & $802.11 \mathrm{~b}$ & IrDA & IrDA & $802.11 \mathrm{~b}$ & $802.11 \mathrm{~b}$ \\
\hline Slider Setting 4 & $802.11 \mathrm{~b}$ & $802.11 \mathrm{~b}$ & Bluetooth & Bluetooth & $802.11 \mathrm{~b}$ & $802.11 \mathrm{~b}$ \\
\hline Slider Setting 5 & IrDA & IrDA & IrDA & IrDA & IrDA & IrDA \\
\hline Slider Setting 6 & Bluetooth & Bluetooth & Bluetooth & Bluetooth & Bluetooth & Bluetooth \\
\hline Slider Setting 7 & $802.11 \mathrm{~b}$ & $802.11 \mathrm{~b}$ & $802.11 \mathrm{~b}$ & $802.11 \mathrm{~b}$ & $802.11 \mathrm{~b}$ & $802.11 \mathrm{~b}$ \\
\hline
\end{tabular}

Fig. 7. Comparison of the selected transport dependant on various slider settings and priority schemes.

affect transport selection, 2) tips on setting up the user profile and 3) a default profile for users that may not want to interact directly with QoT.

\section{A. Examining Profiles}

Transport selection depends on the slider settings and the priority order. The preferred transport can change with slight variations to either of these elements. Using the iPAQ specification data from Figure 4, Figure 7 shows the transport that PSCS selects for seven slider settings combined with all six possible prioritization schemes.

Due to space limitations, a textual description of the slider settings is provided in lieu of graphical representations. Slider Setting 1 sets the green slider on SPEED to 4 Mbps and the red slider to $115 \mathrm{Kbps}$. On the POWER bar, the green slider is set to $4 \mathrm{dBm}$ and the red slider is not set. On the RANGE bar, the green slider is set to $10 \mathrm{~m}$ and the red slider is not set. Slider Setting 2 moves the red slider on POWER to $17 \mathrm{dBm}$. Subsequently, gray zones appear on SPEED up to 4 Mbps and on RANGE up to $10 \mathrm{~m}$. Slider Setting 3 is identical to Slider Setting 1 except that the green slider on RANGE is moved up to $333 \mathrm{~m}$. Slider Setting 4 is identical to Slider Setting 3 except that the red slider on RANGE is set to $1.7 \mathrm{~m}$, with a gray zone appearing on POWER up to $4 \mathrm{dBm}$. Slider Setting 5 is identical to Slider Setting 3 except that the red slider on POWER is set to $17 \mathrm{dBm}$. Gray zones appear on SPEED up to $4 \mathrm{Mbps}$ and on RANGE up to $10 \mathrm{~m}$. Slider Setting 6 combines Slider Settings 4 and 5. Slider Setting 7 is the same as Slider Setting 4 with the red slider on SPEED changed to 1 Mbps and an additional gray zone appearing on RANGE up to 366 $\mathrm{m}$.

In row 1 of Figure 7 all 3 transports are represented and are selected based on a combination of their strengths. IrDA is selected when speed and power are important, while WiFi is chosen to achieve speed and range and Bluetooth is selected when power and range are high priorities.

Rows 2-4 effectively reduce availability from 3 to 2 transports, allowing us to compare transport preferences in a pairwise fashion.

Rows 5-7 establish IrDA, Bluetooth and WiFi as the dominant choices respectively since each of the other two transports is either unacceptable or undesirable. 
Overall, these comparisons demonstrate that relatively small changes to the slider or the prioritization settings can have a significant effect on the selection of the best transport.

\section{B. Profile Guidelines}

The user profile settings can be somewhat sensitive. Consequently, we suggest seven guidelines for setting up a user profile. Examples are taken from the transport set in Figure 4.

1) Using the red slider eliminates some transports from consideration. For example, in Figure 2 the red slider for POWER, at $17 \mathrm{dBm}$, eliminates $802.11 \mathrm{~b}$ from the available options.

2) If a criterion is all green (all-inclusive), then the priority number does not matter. For example, setting the green slider on RANGE to $0 \mathrm{~m}$ as in Figure 8 creates a onebranch search tree for the range priority.

3) If a criterion is set to include only one possibility (allrestrictive), then the priority number does not matter. For example, in Figure 8 setting the COST red slider next to $\$ 0$ creates a one-branch search tree on cost with the only option being $\$ 0$.

4) To maximize device performance for a particular criterion, the green slider should be set high. For example, if speed is of paramount importance, then setting the green slider high, as in Figure 2, will attempt to compromise on any lower priority criteria in order to select the highspeed $802.11 \mathrm{~b}$ transport.

5) If the green slider is set too high and the best option fails, then the prioritization falls to the next tier and the upper priority is virtually useless (except in tiebreaking). For example, in the previous example, if $802.11 \mathrm{~b}$ is unavailable (the best option on speed), or a high throughput is not achieved, then anything else is acceptable under the yellow range and the lower priority criteria become the decisive factors.

6) To get a combination of priorities, the green slider should be set mid-range. For example, by setting the green sliders on SPEED and POWER to include half the range of values as desirable options, as in Figure 8, a speed/power combination results.

7) If the green slider is set too low on a high priority, then the lower priorities will be asserted. For example, if the POWER and RANGE settings both favor Bluetooth, then even if speed is the top priority, setting the green slider below .7 Mbps will likely select Bluetooth as the preferred transport, which has relatively poor speed.

\section{Default Profile}

The PSCS default profile shown in Figure 8 is established following the above guidelines.

The first priority is cost. The COST bar is all-restrictive, allowing only $\$ 0$ connections. The all-restrictive condition is necessary since we can't assume the user is willing to pay connection charges unless specifically set by the user.

Speed is set as priority 2 . The SPEED bar is half green, half yellow. This provides a good generic setting that will yield a combined speed/power selection.

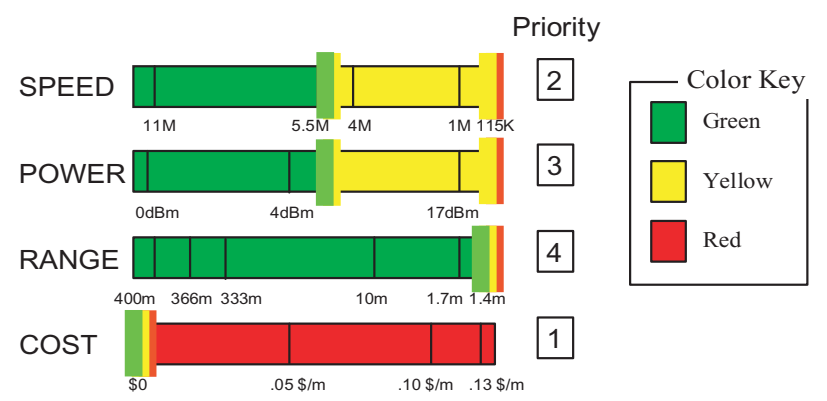

Fig. 8. An example of a default user profile for PSCS.

Power is set as priority 3 with the POWER bar also set to half green and half yellow. PSCS also defaults to automatically observe the remaining battery life of the device. Anytime the battery life drops below 25\%, power is changed to priority 1 .

The lowest priority is range. The RANGE bar is all-inclusive to allow QoT to use any transport available.

\section{CONCLUSION}

Utilizing heterogeneous transport switching via QoT and intelligent transport selection via PSCS, a satisfying connection to another device can be established and maintained despite changes to the environment or user preferences. This paper presented the methodology for PSCS, including its advantageous ability to present viable options, criteria tradeoff and measurement feedback. Equations for determining tradeoff limitations were given and shown to have a tighter bound than the established theoretical equations. Recommendations for setting the user profile were also set forth and a generic default profile was provided.

\section{REFERENCES}

[1] M. Baker, X. Zhao, and J. Stone, "Supporting mobility in MosquitoNet." Proceedings of the 1996 USENIX Technical Conference, Jan. 1996, pp. 120-127.

[2] P.M.L. Chan, Y.F. Hu and R.E. Sheriff. "Implementation of fuzzy multiple objective decision making algorithm in a heterogeneous mobile environment." Proceedings of the IEEE Wireless Communications and Networking Conference, Orlando, FL, USA, March 2002, pp. 17-21.

[3] M. Stemm and R.H. Katz, "Vertical handoffs in wireless overlay networks." ACM Mobile Networking and Applications, no. 4. ACM, 1999, pp. 335-350.

[4] H. Wang, R.H. Katz, and J. Giese. "Policy-enabled handoff across heterogeneous wireless networks." 2nd IEEE Workshop on Mobile Computing and Applications (WMCSA 1999), New Orleans, February 1999.

[5] J.C. Funk, H.R. Duffin, L. Dai, C.D. Knutson. "Inverse multiplexing in short-range multi-transport wireless communications." Proceedings of the IEEE Wireless Communications and Networking Conference 2003 (WCNC 2003), New Orleans, Louisiana, March 17-19, 2003.

[6] C.D. Knutson, S.B. Barnes, R.W. Woodings, H.D. Duffin, J.M. Brown. "Quality of Transport (QoT): Dynamic Autonomous Transport Selection in Heterogeneous Wireless Environments." Proceedings of the IEEE Wireless Communications and Networking Conference 2004 (WCNC 2004), Atlanta, Georgia, March 21-25, 2004.

[7] S.B. Barnes, R.W. Woodings, C.D. Knutson. "Transport Discovery in Wireless Multi-Transport Environments." Proceedings of the IEEE Wireless Communications and Networking Conference 2003 (WCNC 2003), New Orleans, Louisiana, March 17-19, 2003.

[8] D. Johnson and D. Maltz. "Protocols for adaptive wireless and mobile networking." IEEE Personal Communications, 3(1):34-42, Feb. 1996.

[9] K. Chebrolu and R. Rao. "Communication using multiple wireless interfaces." Proceedings of the IEEE Wireless Communications and Networking Conference 2002 (WCNC 2002), Orlando, Florida, March 18-21, 2002. 